\title{
Rear shock formation in gravity currents
}

\author{
S. J. D. D’Alessio ${ }^{1}$, J. P. Pascal ${ }^{2}$ \& T. B. Moodie ${ }^{3}$ \\ ${ }^{1}$ Department of Applied Mathematics, University of Waterloo, Canada \\ ${ }^{2}$ Department of Mathematics, Ryerson University, Canada \\ ${ }^{3}$ Department of Mathematical and Statistical Sciences, \\ University of Alberta, Canada
}

\begin{abstract}
Considered in this study is the gravity driven flow of a two-fluid system arising from the motion of a heavy fluid in a rectangular channel having a flat bottom. The mathematical model is based on shallow-water theory in connection with a two-layer Boussinesq fluid. By means of a scaling argument, it can be shown that for small density differences the gravity current can be successfully modelled by a two-by-two hyperbolic system in conservation form together with a pair of algebraic relations. This reduced system is referred to as the weak stratification model. A weakly nonlinear analysis is performed on this weak stratification model to elicit information concerning the formation of a rear shock which may form on the back side of the head of the gravity current. Predictions made by the analytical technique are then verified by numerical simulations.

Keywords: shallow-water theory, Boussinesq approximation, two-layer model, hyperbolic system, multiple scales analysis.
\end{abstract}

\section{Introduction}

A gravity current refers to the flow of one fluid within another which is driven by the density difference between these two fluids. Gravity currents play an important role in many known natural phenomena as well as human-related activities ranging from turbidity currents to the accidental release of industrial pollutants.

Although it is obviously the case that the initial flow following the release of a gravity current of finite volume is a complex three-dimensional unsteady flow, soon after release the current will have spread sufficiently that its length is very much greater than its thickness. The thickness $h(x, t)$ will at this stage be slowly 


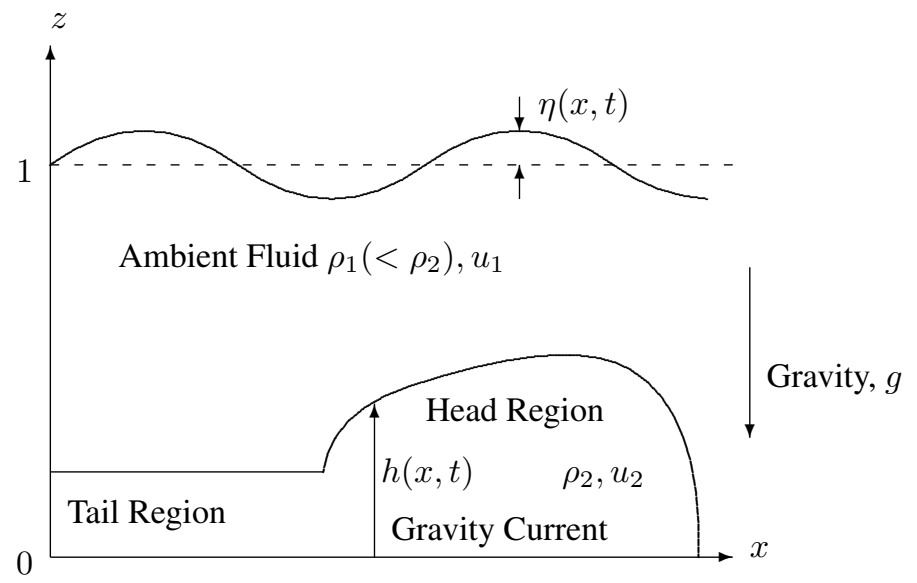

Figure 1: The flow configuration of the two-fluid system and the general structure of a bottom gravity current.

varying over the horizontal position $x$ and in time $t$. This approach to gravity currents has been exploited successfully by numerous researchers in the past and we refer the reader to the book by Simpson [1] for an extensive bibliography and comparisons between theory based on this low aspect ratio approach and experiments. The general structure of a bottom gravity current is shown in Figure 1.

A distinguished feature of these flows, which is the focus of this study, is the formation of a rear shock behind the head of the gravity current. Experiments executed by Rottman and Simpson [2] examined instantaneous releases for $0<h_{i} \leq 1$, where $h_{i}$ is the initial depth ratio between the released heavy fluid and the total depth of the two-fluid system in the rectangular channel. Their observations revealed that for $h_{i}$ equal to or slightly less than unity the disturbance generated at the proximal end wall has the appearance of an internal hydraulic drop. On the other hand, for smaller values of $h_{i}(\lesssim 0.7)$ this disturbance is a long wave of depression. Currently there are no theoretical model-based calculations that can accurately predict this bifurcation in behaviour which occurs in the experimental results as $h_{i}$ is varied. These experiments did however serve to emphasize the importance of including the effects of the ambient fluid on the bottom boundary current when the current initially occupies a large fraction of the total depth.

D'Alessio et al [3] employed a two-layer shallow-water model to study bottom gravity currents released from rest. Using MacCormack's method [4] to integrate numerically the hyperbolic system they were able to achieve good qualitative agreement with the experimental results of Rottman and Simpson [2]. Also, employing multiple scales arguments they were able to show analytically the dependence of internal bore formation (i.e. rear shock) on initial fractional depth of the release volume. Their analysis, however, did not confirm the value of $h_{i} \simeq 0.7$ referred to earlier but rather gave the lower value of $h_{i}=0.5$ as the minimum 
fraction for bore initiation. This is perhaps not surprising when one contrasts the relative simplicity of a shallow water (hydraulic) model for what is a complex flow involving possible nonhydrostatic effects in various regions of the flow due to streamline curvature, unresolved small scale dissipation and other effects.

The goal of the present work is to extend the analysis in [3] to include bottom gravity currents released with an arbitrary constant initial velocity in a rectangular channel.

\section{Formulation}

The flow configuration and general structure of the gravity current is depicted in Figure 1. Here, $\eta(x, t)$ represents the dimensionless displacement of the free surface from its undisturbed height, $\left(u_{1}, u_{2}\right)$ are the dimensionless fluid velocities in Cartesian coordinates $(x, z)$. In dimensionless form the mean depth of the two layer system measured from $z=0$ is taken to be unity, and $h(x, t)$ is the dimensionless fractional thickness of the bottom layer (or gravity current). The flat bottom of the rectangular channel is located at $z=0$. The flow is driven by the buoyancy force arising because of the difference between the density $\rho_{2}$ of the bottom layer and the density $\rho_{1}$ of the ambient fluid.

In dimensionless variables the governing shallow-water equations take the form:

$$
\begin{gathered}
\frac{\partial u_{1}}{\partial t}+u_{1} \frac{\partial u_{1}}{\partial x}+\frac{\partial \eta}{\partial x}=0 \\
\frac{\partial}{\partial t}\left(h-\frac{g^{\prime}}{g} \eta\right)+\frac{\partial}{\partial x}\left[\left(1+\frac{g^{\prime}}{g} \eta-h\right) u_{1}\right]=0 . \\
\frac{\partial u_{2}}{\partial t}+u_{2} \frac{\partial u_{2}}{\partial x}+\left(1-\frac{g^{\prime}}{g}\right) \frac{\partial \eta}{\partial x}+\frac{\partial h}{\partial x}=0 \\
\frac{\partial h}{\partial t}+\frac{\partial}{\partial x}\left(h u_{2}\right)=0 .
\end{gathered}
$$

In the above $g^{\prime}=g\left(\rho_{2}-\rho_{1}\right) / \rho_{2}$ is the reduced gravity and the parameter $g^{\prime} / g$ is a measure of the stratification of this two-fluid system. As explained in [3], it is also a measure of the importance of the free surface on the flow since letting $g^{\prime} / g \rightarrow 0$ filters out surface wave phenomena. The system of equations (1)-(4) is posed as an initial value problem subject to the initial conditions

$$
u_{1}(x, 0)=0, u_{2}(x, 0)=u_{20}, \eta(x, 0)=0, h(x, 0)=G(x),
$$

the impermeability conditions

$$
u_{1}(0, t)=0, u_{2}(0, t)=0,
$$

the slope conditions

$$
\frac{\partial \eta}{\partial x}(0, t)=\frac{\partial h}{\partial x}(0, t)=0,
$$


and lastly the far-field conditions

$$
u_{1}(x, t) \rightarrow 0, u_{2}(x, t) \rightarrow 0, \eta(x, t) \rightarrow 0, h(x, t) \rightarrow 0 \text { as } x \rightarrow \infty .
$$

In the above $G(x)$ specifies the initial configuration of the two-fluid system. We are particularly interested in initial rectangular configurations of the form

$$
G(x)=\left\{\begin{array}{ll}
h_{0} & \text { if } 0 \leq x \leq x_{0} \\
0 & \text { if } x>x_{0}
\end{array},\right.
$$

where $h_{0}$ is the nondimensional initial thickness of the gravity current and $u_{20}$ is its corresponding initial velocity. The parameter $h_{0}$ is thus the ratio of the initial depth of the heavy fluid to that of the two-fluid system.

An important simplified model is the weakly stratified model wherein we neglect terms of $O\left(g^{\prime} / g\right)$ on the assumption that the initial density difference is small. It has been shown in [3] that in this limit the governing equations can be reduced to the two-by-two system

$$
\begin{gathered}
\frac{\partial u_{2}}{\partial t}+\left(u_{2}+\frac{\partial \eta}{\partial u_{2}}\right) \frac{\partial u_{2}}{\partial x}+\left(1+\frac{\partial \eta}{\partial h}\right) \frac{\partial h}{\partial x}=0, \\
\frac{\partial h}{\partial t}+\frac{\partial}{\partial x}\left(h u_{2}\right)=0,
\end{gathered}
$$

together with the two algebraic relations given by

$$
\begin{gathered}
\eta=\eta\left(u_{2}, h\right)=-\frac{u_{2}^{2} h}{1-h}-\frac{1}{2} h^{2}, \\
u_{1}=-\frac{h u_{2}}{1-h} .
\end{gathered}
$$

Alternatively, the above can be expressed more compactly as

$$
\begin{gathered}
\frac{\partial u_{2}}{\partial t}+\frac{(1-3 h)}{(1-h)} u_{2} \frac{\partial u_{2}}{\partial x}+\left(1-h-\frac{u_{2}^{2}}{(1-h)^{2}}\right) \frac{\partial h}{\partial x}=0, \\
\frac{\partial h}{\partial t}+\frac{\partial}{\partial x}\left(h u_{2}\right)=0 .
\end{gathered}
$$

Another more simplified model worth mentioning is the weakly stratified deep ambient layer model given by

$$
\begin{gathered}
\frac{\partial u_{2}}{\partial t}+u_{2} \frac{\partial u_{2}}{\partial x}+\frac{\partial h}{\partial x}=0, \\
\frac{\partial h}{\partial t}+\frac{\partial}{\partial x}\left(h u_{2}\right)=0 .
\end{gathered}
$$

This model applies when $h \ll 1$.

In the next section we carry out a multiple scales analysis on the weakly stratified model equations to elicit information regarding rear shock formation. 


\section{Weakly nonlinear analysis}

In this section we will analytically investigate the formation of the rear shock behind the head of the gravity current. Since shock formation is a nonlinear phenomenon, we employ a weakly nonlinear analysis, similar to that in [3], on the weakly stratified model equations given by (14) and (15). We first expand the flow variables about the basic state given by $(u, h)=\left(u_{0}, h_{0}\right)$, taking $u \equiv u_{2}$, which corresponds to the initial configuration. The weakly stratified model equations can then be reduced to the following quadratically nonlinear system:

$$
\begin{gathered}
\frac{\partial \hat{u}}{\partial t}+\left(\frac{u_{0}\left(1-3 h_{0}\right)}{\left(1-h_{0}\right)}+\frac{\left(1-3 h_{0}\right)}{\left(1-h_{0}\right)} \hat{u}-\frac{2 u_{0}}{\left(1-h_{0}\right)^{2}} \hat{h}\right) \frac{\partial \hat{u}}{\partial x} \\
+\left(\frac{\left(1-h_{0}\right)^{3}-u_{0}^{2}}{\left(1-h_{0}\right)^{2}}-\frac{2 u_{0}}{\left(1-h_{0}\right)^{2}} \hat{u}-\frac{\left[\left(1-h_{0}\right)^{3}+2 u_{0}^{2}\right]}{\left(1-h_{0}\right)^{3}} \hat{h}\right) \frac{\partial \hat{h}}{\partial x}=0, \\
\frac{\partial \hat{h}}{\partial t}+\left(h_{0}+\hat{h}\right) \frac{\partial \hat{u}}{\partial x}+\left(u_{0}+\hat{u}\right) \frac{\partial \hat{h}}{\partial x}=0,
\end{gathered}
$$

where the hat denotes the deviation from the basic state $\left(u_{0}, h_{0}\right)$.

Linearizing the above equations and assuming a wave-like solution

$$
u(x, t)=u(\xi), h(x, t)=h(\xi) \text { where } \xi=x-c t
$$

(dropping the hats) we find that the linearized speeds

$$
c_{ \pm}=\left(\frac{1-2 h_{0}}{1-h_{0}}\right) u_{0} \pm \sqrt{\frac{h_{0}}{1-h_{0}}} \sqrt{\left(1-h_{0}\right)^{2}-u_{0}^{2}},
$$

guarantee a nontrivial solution. For $0 \leq u_{0} \leq 1$ it is clear that the speeds are real in the triangular region $h_{0} \leq 1-u_{0}$.

Equations (18) and (19) can be combined to yield a single equation given by (again dropping the hats)

$$
h_{t t}+a_{1} h_{x t}+a_{2} h_{x x}=-(u h)_{x t}+a_{3}\left(u u_{x}\right)_{x}-a_{4}\left(h h_{x}\right)_{x}-a_{5}(u h)_{x x},
$$

with subscripts denoting partial differentiation and

$$
\begin{gathered}
a_{1}=\frac{2 u_{0}\left(1-2 h_{0}\right)}{\left(1-h_{0}\right)}, a_{2}=\frac{u_{0}^{2}\left(1-3 h_{0}+3 h_{0}^{2}\right)-h_{0}\left(1-h_{0}\right)^{3}}{\left(1-h_{0}\right)^{2}}, \\
a_{3}=\frac{h_{0}\left(1-3 h_{0}\right)}{\left(1-h_{0}\right)}, a_{4}=\frac{h_{0}\left[\left(1-h_{0}\right)^{3}+2 u_{0}^{2}\right]}{\left(1-h_{0}\right)^{3}}, a_{5}=\frac{u_{0}\left(1-2 h_{0}+3 h_{0}^{2}\right)}{\left(1-h_{0}\right)^{2}} .
\end{gathered}
$$

We next introduce

$$
\xi=x-c_{-} t, \eta=x+c_{-} t, T=\varepsilon t, h=\varepsilon \tilde{h}, u=\varepsilon \tilde{u} .
$$


In addition, we expand the variables in the following series

$$
\tilde{h}=h^{(0)}+\varepsilon h^{(1)}+O\left(\varepsilon^{2}\right) \text { and } \tilde{u}=u^{(0)}+\varepsilon u^{(1)}+O\left(\varepsilon^{2}\right) .
$$

The leading order equations then become

$$
\begin{gathered}
\alpha h_{\eta \eta}^{(0)}-\beta h_{\eta \xi}^{(0)}=0, \\
c_{-}\left(u_{\eta}^{(0)}-u_{\xi}^{(0)}\right)+\frac{u_{0}\left(1-3 h_{0}\right)}{\left(1-h_{0}\right)}\left(u_{\eta}^{(0)}+u_{\xi}^{(0)}\right)= \\
-\frac{\left[\left(1-h_{0}\right)^{3}-u_{0}^{2}\right]}{\left(1-h_{0}\right)^{2}}\left(h_{\eta}^{(0)}+h_{\xi}^{(0)}\right),
\end{gathered}
$$

with $\alpha=c_{-}^{2}+a_{1} c_{-}+a_{2}$ and $\beta=2\left(c_{-}^{2}-a_{2}\right)$. The solutions have the form

$$
\begin{gathered}
h^{(0)}=\phi(\xi, T)+\psi\left(\eta+\frac{\alpha}{\beta} \xi, T\right), \\
u^{(0)}=\frac{\Gamma}{\gamma} \phi(\xi, T)-\frac{\left(1+\frac{\alpha}{\beta}\right) \Gamma}{\omega} \psi\left(\eta+\frac{\alpha}{\beta} \xi, T\right),
\end{gathered}
$$

where $\phi$ and $\psi$ are arbitrary functions and

$$
\begin{gathered}
\Gamma=\frac{\left[\left(1-h_{0}\right)^{3}-u_{0}^{2}\right]}{\left(1-h_{0}\right)^{2}}, \gamma=c_{-}-\frac{u_{0}\left(1-3 h_{0}\right)}{\left(1-h_{0}\right)}, \\
\omega=\left(1-\frac{\alpha}{\beta}\right) c_{-}+\left(1+\frac{\alpha}{\beta}\right) \frac{\left(1-3 h_{0}\right) u_{0}}{\left(1-h_{0}\right)} .
\end{gathered}
$$

As we will shortly see, of importance to our analysis is the function $\phi$.

Carrying the analysis to the next order enables us to find the correction $h^{(1)}$. After some algebra the following equation for $h^{(1)}$ emerges

$$
\alpha h_{\eta \eta}^{(1)}-\beta h_{\eta \xi}^{(1)}=A(\xi, T)+B(\xi, \eta, T),
$$

where

$$
A(\xi, T)=\left(2 c_{-}-a_{1}\right) \phi_{T \xi}+\frac{s}{2}\left(\phi^{2}\right)_{\xi \xi}
$$

with $s=2 \Gamma\left(c_{-}-a_{5}\right) / \gamma+\Gamma^{2} a_{3} / \gamma^{2}-a_{4}$. To ensure that $h^{(1)}$ remains bounded as $\xi, \eta \rightarrow \pm \infty$ we impose $A=0$ as a solvability condition. We note that the function $B$ does not enter into the analysis. 


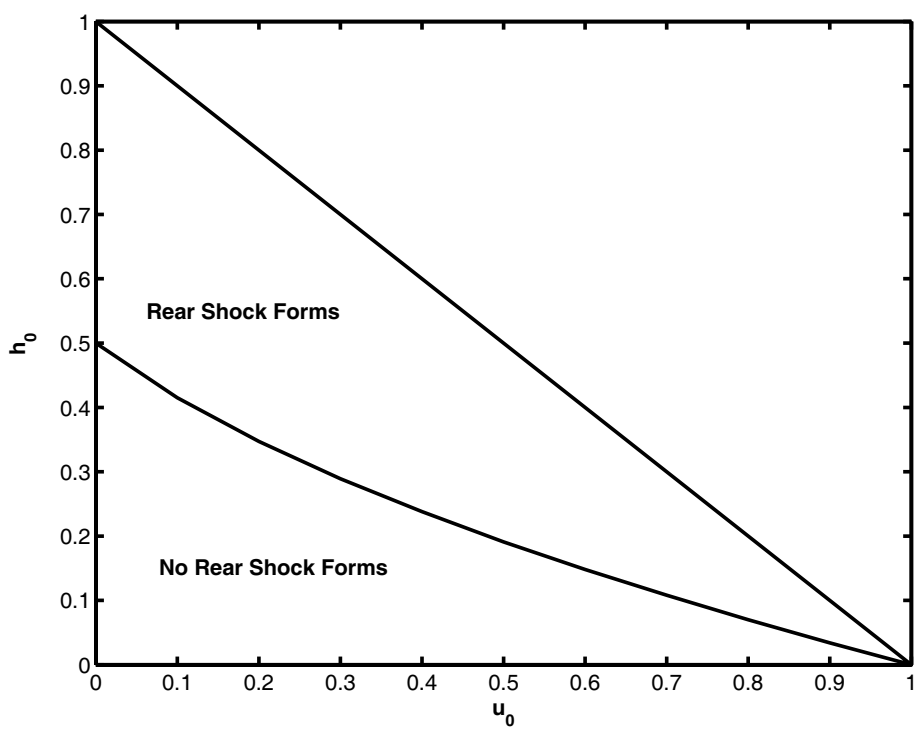

Figure 2: Analytical predictions showing initial configurations which should result in a rear shock.

Integrating $A=0$ with respect to $\xi$ gives

$$
\phi_{T}+b \phi \phi_{\xi}=0, b=\frac{s}{2 c_{-}-a_{1}},
$$

where it was assumed that $\phi$ has compact support. If we let $\phi(\xi, 0)=f(\xi)$ represent the initial condition, then the solution to the above can be expressed implicitly in parametric form in terms of the parameter $\tau$ as

$$
\phi(\xi, T)=f(\tau) \text { along } \xi=b T f(\tau)+\tau .
$$

Shock formation occurs when $\left|\phi_{\xi}\right| \rightarrow \infty$ where

$$
\phi_{\xi}=\frac{f^{\prime}(\tau)}{1+b T f^{\prime}(\tau)},
$$

which becomes infinite when $T=-1 / b f^{\prime}(\tau)$. Along the back side of a smooth curve $f(\tau)$, where $f^{\prime}(\tau)>0$, a shock will form if $b<0$. In terms of the initial configuration specified by $u_{0}$ and $h_{0}$, with $u_{0}$ replacing $u_{20}$ in equation (5), this condition can be expressed as

$$
2 F_{1} F_{2} F_{3}+F_{1}^{2} F_{4}-F_{2}^{2} F_{5}<0,
$$

where

$$
F_{1}=\frac{\left(1-h_{0}\right)^{3}-u_{0}^{2}}{\left(1-h_{0}\right)^{2}}, F_{2}=\frac{u_{0} h_{0}}{1-h_{0}}+\sqrt{\frac{h_{0}}{1-h_{0}}} \sqrt{\left(1-h_{0}\right)^{2}-u_{0}^{2}},
$$




$$
\begin{gathered}
F_{3}=-\frac{u_{0} h_{0}\left(1+h_{0}\right)}{\left(1-h_{0}\right)^{2}}+\sqrt{\frac{h_{0}}{1-h_{0}}} \sqrt{\left(1-h_{0}\right)^{2}-u_{0}^{2}}, F_{4}=\frac{h_{0}\left(1-3 h_{0}\right)}{\left(1-h_{0}\right)}, \\
F_{5}=\frac{h_{0}\left[\left(1-h_{0}\right)^{3}+2 u_{0}^{2}\right]}{\left(1-h_{0}\right)^{3}} .
\end{gathered}
$$

A plot of the region satisfying the above inequality is shown in Figure 2. As a check, if we set $u_{0}=0$ then the above condition collapses to simply $h_{0}>1 / 2$ which is in full agreement with our previous result reported in [3].

We conclude this section by mentioning that if the above analysis is repeated on the weakly stratified deep ambient layer model equations (16)-(17), the prediction is that a rear shock should always form. This result, however, is not consistent with our numerical simulations. Thus, the further simplifications inherent in these equations render them inadequate in capturing the essential physics of the flow.

\section{Numerical results and discussion}

We next discuss the technique used to numerically integrate the weakly stratified equations. The goal here is to validate the analytical predictions derived in the previous section.

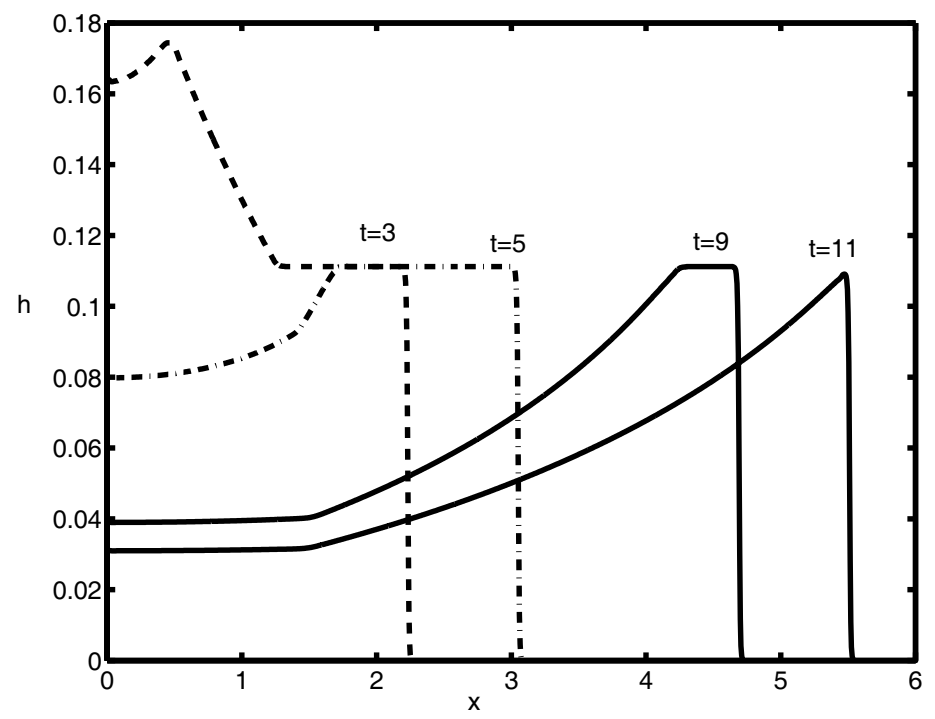

Figure 3: The evolution of the gravity current with $x_{0}=1, h_{0}=0.3$ and $u_{0}=0$.

In order to obtain numerical solutions to the weakly stratified equations we employed the SLIC method which is a conservative high-order TVD scheme [5]. Based on the MUSCL-Hancock approach, second-order accuracy is obtained by 
considering a piecewise linear reconstruction of the cell-averaged approximations over computational cells of the spatial domain. A slope limiter is applied in order to obtain non-oscillatory results.

The cell-averaged approximate solution is updated at the subsequent time level by a finite-volume scheme. The numerical flux employed is the FORCE flux, which is given by the arithmetic mean between the Lax-Friedrichs flux and the two-step Lax-Wendroff flux. The resulting scheme is thus centred and as such does not require the information provided by the decomposition of the Jacobian of the flux vector into characteristic fields which is essential for upwind based methods.

Shock formation can be determined from the numerical solution by examining the solution for $h(x, t)$ as a function of $x$ for a fixed value of $t$. This distribution reveals the structure of the gravity current at a particular time. In Figure 3 we present the evolution of the gravity current resulting from the release from rest of a fixed volume of fluid with $h_{0}=0.3$. As expected, due to the low value of $h_{0}$ the disturbance generated at the proximal end wall evolves into a long wave of depression on the back side of the head of the gravity current.

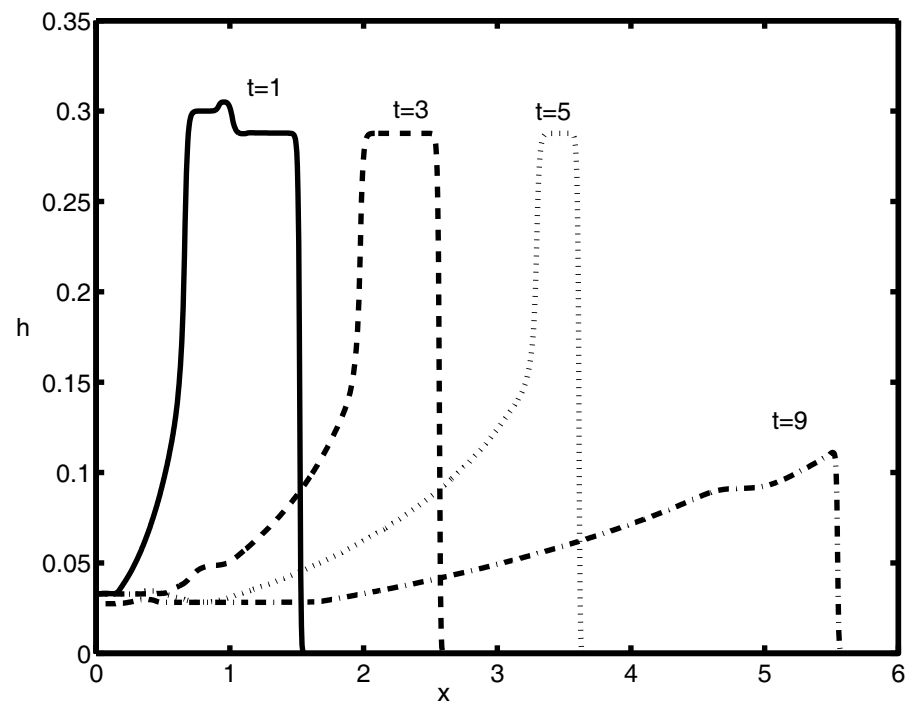

Figure 4: The evolution of the gravity current with $x_{0}=1, h_{0}=0.3$ and $u_{0}=0.5$.

The evolution presented in Figure 4 indicates that with the same initial depth ratio of $h_{0}=0.3$, an initial velocity of $u_{0}=0.5$ is sufficiently large to generate a gravity current exhibiting the formation of a rear shock. To illustrate the dependence of the generation of a rear shock on the initial velocity of the heavy fluid, in Figure 5 we display the structure of the gravity current corresponding to different values of $u_{0}$ at a fixed time. It can clearly be seen that as $u_{0}$ increases the back 


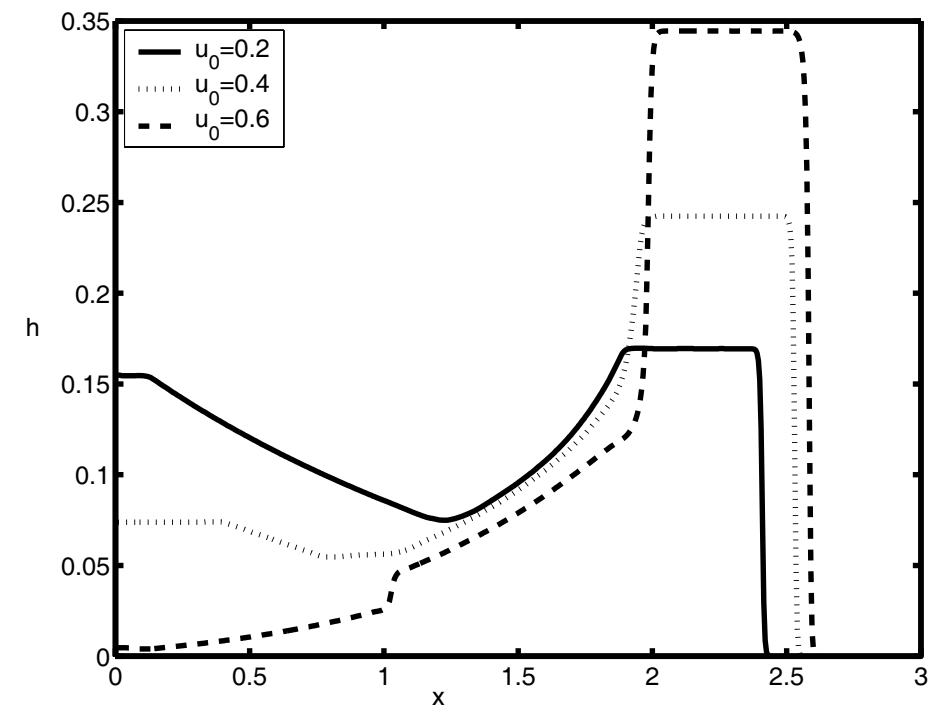

Figure 5: The structure of the gravity current with $x_{0}=1$ and $h_{0}=0.3$ at $t=3$.

side of the head of the gravity current steepens. Our numerical experiments indicate that the critical initial velocity for the formation of the rear shock is in good agreement with the analytical prediction for various values of $h_{0}$.

\section{Concluding remarks}

Discussed in this paper are bottom gravity currents flowing on a flat bottom of a rectangular channel. In particular, the interest here was on the formation of a rear shock formed behind the head of the gravity current. Under conditions of weak stratification a simplified model has been constructed and is amenable to analytical treatment. A weakly nonlinear analysis was successful in predicting when a rear shock should form. These predictions were confirmed by extensive numerical experiments.

\section{Acknowledgements}

Financial support for this research was provided by the Natural Sciences and Engineering Research Council of Canada.

\section{References}

[1] Simpson, J.E., Gravity Currents: In the Environment and the Laboratory, 2nd Ed., Cambridge University Press, Cambridge, UK, 1997. 
[2] Rottman, J.W., \& Simpson, J.E., Gravity currents produced by instantaneous releases of a heavy fluid in a rectangular channel, J. Fluid Mech. 135, pp. 95110, 1983.

[3] D’Alessio, S.J.D., Moodie, T.B., Pascal, J.P., \& Swaters, G.E., Gravity currents produced by sudden release of a fixed volume of heavy fluid, Stud. Appl. Math. 96, pp. 359-385, 1996.

[4] Le Veque, R., Numerical Methods for Conservation Laws, Birkhäuser, Basel, Switzerland, 1992.

[5] Toro, E.F., Riemann Solvers and Numerical Methods for Fluid Dynamics, Springer, Berlin, Germany, 1999. 